\title{
Características radiográficas de los quistes dentígeros diagnosticados en la Facultad de Estomatología de la Universidad Peruana Cayetano Heredia
}

\author{
Radiographics characteristics of dentigerous cysts diagnosed in the Universidad Peruana Cayetano Heredia
}

Maria Alejandra Olaechea-Ramos ${ }^{1, a, b}$, Alexis Evangelista-Alva ${ }^{1, \mathrm{c}}$, Milushka Miroslava Quezada-Márquez ${ }^{1, a, d}$

\section{RESUMEN}

Objetivo: Evaluar las características radiográficas de los quistes dentígeros (QD) diagnosticados en la Facultad de Estomatología de la Universidad Peruana Cayetano Heredia (UPCH). Periodo 2010-2017. Material y métodos: Estudio retrospectivo, descriptivo, observacional y transversal, donde se seleccionaron las radiografías panorámicas de 37 casos de QD. Resultados: El total de casos diagnosticados de quistes dentígeros fue de 233, que luego de los criterios de inclusión y exclusión, se obtuvieron 37 casos, de los cuales, el 45,9\% de casos se encontraron en la segunda década de vida, siendo más frecuente en mujeres $(51,4 \%)$ con una predilección mandibular (59,5\%). Radiolúcidos (97,3\%), de limites definidos (67,6\%), bordes corticalizados $(54,1 \%)$ y uniloculares $(94,6 \%)$. Todos los quistes dentígeros se asociaban a una pieza dental, de las cuales se observaron ápice cerrado $(48,6 \%)$ y desplazamiento dental $(59,5 \%)$. El $56,8 \%$ de casos no se vio afectada la pieza denta 1 adyacente, pero si su lámina dura $(59,5 \%)$. El 68,2\% de casos no afectaron la cortical de la basal mandibular, pero si desplazaron el conducto dentario inferior $(54,5 \%)$ y el $46,7 \%$ de casos desplazaron el piso del seno maxilar. Conclusiones: La mayoría de resultados obtenidos sobre las características en población peruana, apoyan estudios previos reportados en América, Europa y Asia. Radiográficamente el quiste dentígero mostró características que avalan su comportamiento netamente benigno.

PALABRAS CLAVE: Quistes odontogénicos, quiste dentígero, radiografía panorámica. 


\section{SUMMARY}

Objective: To evaluate the x-rays characteristics of dentigerous cysts diagnosed in the Universidad Peruana Cayetano Heredia between 2010 and 2017. Material and Methods: Retrospective, descriptive, observational, and transversal study based on selected panoramic x-rays of dentigerous cysts cases. Results: Only 37 out of a total sample of 237 dentigerous cysts cases were examined based on an inclusion and exclusion criteria. From this sample, 45.9 percent of cysts were found in the second decade of life with a higher frequency of 51.4 percent for women, and a jaw predilection of 59.5\% in all cases. In addition, it was found that 97.3 percent of the cases were radiolucent, defined limits were found in 67.6 percent of cases, corticalized edges in 54.1 percent of cases, and unilocular in 94.6 percent of cases. All dentigerous cysts were associated with a dental piece of which 48.6 percent had a closed apex and dental displacement was observed in 59.5 percent of cases. In 56.8 percent of cases, the adjacent tooth was not affected, but its hard film was affected in 59.5 percent of cases. Moreover, 68.2 percent of the dentigerous cysts did not affect the cortex of the mandibular basal, but 54.5 percent displaced the inferior dental canal and $46.7 \%$ displaced the maxillary sinus floor. Conclusions: Most of the results obtained about characteristics in Peruvian population support previous studies reported in America, Europe and Asia. Radiographically, dentigerous cysts displayed characteristics that support their clearly benign behavior.

\section{KEY WORDS: Odontogenic cysts, dentigerous cyst, radiography panoramic.}

\section{INTRODUCCIÓN}

El quiste dentígero (QD) es uno de los quistes odontogénicos más comunes que se presentan en los maxilares $(1,2)$. El término "dentígero" significa "que contiene un diente" esto quiere decir que se relaciona a la corona de dientes ya sean impactados, sumergidos y no erupcionados, adherido a la corona de éstos en la unión cemento adamantina (UCA) y crece por expansión del folículo $(2,3,4)$, aparentemente en ausencia de estímulos inflamatorios por lo que la presencia de una corona dentro del quiste es el signo frecuente de la lesión (4).

El diagnóstico radiológico de un $\mathrm{QD}$ es sencillo, pero hay que saber diferenciarlo de un folículo dental hiperplásico. Según Devi et al., el espacio folicular normal mide de 3 a $4 \mathrm{~mm}$ desde la superficie del esmalte hasta el borde la lesión, cuando se sospecha de un QD éste tiende a medir más de $5 \mathrm{~mm}$ (5).

El QD generalmente crece sin presentar sintomatología pudiendo existir sin ser descubierto por años, por lo que tiende a ser un hallazgo radiográfico durante el descarte de una pieza dental no erupcionada $(1,6)$.

Las células epiteliales que recubren la luz son capaces de producir cambios metaplásicos, por lo que algunos QD pueden progresar a lesiones más agresivas como por ejemplo queratoquiste odontogénico, ameloblastoma, carcinoma mucoepidermoide y carcinoma intraóseo primario $(2,7)$.

El QD es una entidad solitaria, pero se puede presentar múltiple o multifocal en presencia de síndromes o condiciones sistémicas como mucopolisacaridosis, displasia cleidocraneal, síndrome de Gorlin-Goltz (Síndrome nevus vasocelular), Maroteaux-Lamy y síndrome de Klippel Feil. Sin embargo, se han reportado casos de QDs no asociados a condiciones sistémicas, siendo su ubicación la misma que la de un QD solitario $(5,8)$. También se han reportado QD luego del uso prolongado de ciclosporina A y bloqueadores de canales de calcio (5).

El QD se origina por la presión ejercida por un diente en erupción dentro de un folículo impactado, éste obstruye el flujo venoso conduciendo a un rápido transudado de suero a través de las paredes de los capilares sanguíneos, aumentando la presión hidrostática del fluido, lo que ocasiona la separación del folículo de la corona con o sin epitelio reducido del esmalte (5).

Langlais, clasifica a los QDs con respecto a la corona del diente afectado en: quiste de erupción, circunferencial y lateral (9). Existe una cuarta variante llamado "quiste dentígero inflamatorio". El de erupción es una variante del quiste folicular, el cual está cercano a la cresta alveolar. Clínicamente se verá el enrojecimiento, aumento de volumen de la encía, lo cual es 
la expansión de la lesión hacia los tejidos blandos; el color de esta lesión se da por la propiedad refractaria del fluido quístico. El circunferencial ocurre cuando el diente asociado entra en erupción o está entrando en erupción a través de la pared superior del quiste.

En una vista lateral, el quiste aparece en ambos lados de la corona y en última instancia puede rodear las raíces de la pieza asociada. El lateral se ve junto al diente implicado, se desplaza hacia un lado de la corona y cuando el diente intenta erupcionar, se produce esta variante. Cuando esta variante esta hacia distal o cerca de un tercer molar mandibular vertical impactado hay que diferenciarlo de un quiste paradental. La variante inflamatoria surge de la propagación de la inflamación periapical de un diente primario no vital. Esta posibilidad fue reportada por Shaw 1980. La secuela más común de la inflamación periapical no tratada en un diente primario era la penetración intrafolicular de los elementos inflamatorios del diente permanente subyacente. La proliferación subsiguiente del epitelio reducido del esmalte es estimulada por el proceso inflamatorio.

El QD es el segundo quiste más común luego del quiste radicular, representando el $24 \%$ de todos los quistes mandibulares (5). Afectando predominantemente al sexo masculino que el femenino, en una relación 2:1 (10). Las piezas más afectadas son los terceros molares inferiores, caninos maxilares y premolares (2). Clínicamente, hay ausencia dental y áreas de hinchazón, pero sin dolor o molestias, pero si presencia de eritema. Así mismo, a la palpación puede presentarse crepitación debido a la expansión de las corticales óseas, es por ello que el QD puede alcanzar un gran tamaño por lo que veremos al paciente con asimetría facial (11).

Radiográficamente, se presenta como un área radiolúcida bien definida de bordes corticalizados y unilocular con un diámetro mayor a $2,5 \mathrm{~cm}$ alrededor de la corona de un diente no erupcionado. Puede provocar reabsorción radicular de piezas permanentes o deciduas y algunas veces se presenta multilocular con márgenes festoneados o discontinuos $(2,3,6)$, pudiendo ser confundido por otra lesión odontogénica como por ejemplo ameloblastoma uniquístico, queratoquiste, tumor odontogénico adenomatoide (TOA) y fibroma ameloblástico; siendo estos casos oportunos evaluarlos minuciosamente $(4,10)$.
Además, Langlais (9) nos refiere que la variante lateral del QD puede ser confundido con el quiste paradental (QP) siendo sus principales diferencias las siguientes: El QP usualmente se acompaña por un tercer molar parcialmente erupcionado con pericoronitis y el QD lateral se asocia a un diente sin erupcionar, el QP tiene forma semilunar o en llama cuando es pequeño, en cambio el QD lateral es más simétrico y redondeado, el QP raramente crece muchos centímetros en cambio el QD lateral puede ocupar toda la hemimandibula; el margen esclerótico del QP es grueso o espeso, el del QD lateral es delgado y bien definido.

El presente estudio se realizó con la finalidad de evaluar las características radiográficas de los quistes dentígeros (QD) diagnosticados en la Facultad de Estomatología de la Universidad Peruana Cayetano Heredia (UPCH). Periodo 2010-2017.

\section{MATERIAL Y MÉTODOS}

Estudio descriptivo, observacional y transversal. El procedimiento de selección de los casos se inició con la revisión de la base de datos de diagnósticos anatomopatológicos del Laboratorio de Patología Bucomaxilofacial entre enero 2010 y julio del 2017. Luego de ello se revisó la base de datos de radiografías digitales del Servicio de Radiología Bucomaxilofacial de la UPCH.

La población muestral fue seleccionada por conveniencia e incluyó todos los casos diagnosticados en el periodo que cumplan con los criterios de inclusión.

Se obtuvo 37 radiografías panorámicas digitales tomadas con el equipo Orthophos XG 5 de Sirona, el cual opera de $60 \mathrm{Kv}$ a $90 \mathrm{Kv}$ y de $3 \mathrm{~mA}$ a $12 \mathrm{~mA}$, las imágenes digitales fueron exportadas a una computadora compatible Lenovo H61. Para generar las imágenes se utilizó el software Sidexis Next Generation. La revisión de las radiografías se realizó bajo la estricta supervisión de un especialista de Radiología Oral y Maxilofacial con más de 10 años de experiencia.

En cada radiografía panorámica se evalúo la lesión observando las características en estudio.

Se utilizó una ficha donde se registraron las siguientes variables: edad, sexo, localización, densidad, limites, bordes, multilocularidad, pieza dental incluida en la 
lesión, afectación a estructuras anatómicas adyacentes y en 12 casos que presentaban otro tipo de proyecciones se evaluó el compromiso de las tablas óseas.

La metodología planteada para la investigación fue revisada y aprobada por el Comité Institucional de Ética de la UPCH. Cabe recalcar que como la investigación fue una revisión retrospectiva de registros radiográficos no se expusieron a pacientes nuevos a radiaciones ionizantes con fines de investigación.

\section{RESULTADOS}

El total de casos diagnosticados de quistes dentígeros fue de 233, que luego de los criterios de inclusión y exclusión, se obtuvieron 37 casos.

La edad mínima fue de 8 años y la máxima de 75 años, con una edad media de 22,8 años. El quiste dentígero fue más frecuente en mujeres, con 19 casos $(51,4 \%)$, mientras los hombres representaron 18 casos $(48,6 \%)$.

La localización anatómica de las lesiones esta descrita en la tabla 1 y figura 1.

La distribución de las características radiográficas de densidad, límites, bordes y configuración interna se muestran en la tabla 2, figura 2 y figura 3 .
Se observó que los 37 casos (100\%) de quistes dentígeros estaban asociados a una pieza dental no erupcionada y el porcentaje mayor correspondió a las terceras molares $(51,3 \%)$, la distribución de las piezas asociadas se detalla en la tabla 3 y figura 4 . Además, en 17 casos $(45,9 \%)$ se presentó el ápice radicular abierto, en $18(48,6 \%)$ se presentó con ápice abierto y en dos casos no se pudo evaluar el ápice. La afección a piezas dentarias adyacentes y lámina dura se detalla en la tabla 4 y figura 5 .

Los efectos que produce la lesión a las estructuras anatómicas adyacentes en casos del maxilar inferior se muestran en la tabla 5 y figura 5. Adicionalmente, en la muestra hubo 12 casos (100\%) que presentaban otro tipo de radiografías como pósteroanterior, inversa de Towne u oclusal y se pudo registrar el desplazamiento de las tablas óseas, 7 casos $(58,3 \%)$ presentaron desplazamiento de la tabla ósea vestibular, en 3 casos $(25 \%)$ no se vieron afectadas las tablas óseas, en 1 caso $(8,3 \%)$ se vio afectada la tabla lingual y de igual manera en un caso $(8,3 \%)$ se vieron afectadas ambas tablas óseas.

Tabla 1. Distribución según localización de los quistes dentígeros en los maxilares.

\begin{tabular}{lcc}
\hline Localización & $\mathbf{n}$ & $\boldsymbol{\%}$ \\
\hline Superior & 3 & 8,1 \\
Anterior & 1 & 2,7 \\
Posterior & 4 & 10,8 \\
Anterior y Posterior & 7 & 18,9 \\
Posterior y Tuberosidad & & \\
Inferior & 1 & 2,7 \\
Anterior & 12 & 32,4 \\
Posterior & 2 & 5,4 \\
Anterior y Posterior & 1 & 2,7 \\
Posterior Angulo y Rama & 1 & 2,7 \\
Posterior y Angulo & 5 & 13,5 \\
Posterior y rama & & $\mathbf{1 0 0 , 0}$ \\
Total & $\mathbf{3 7}$ & \\
\hline
\end{tabular}




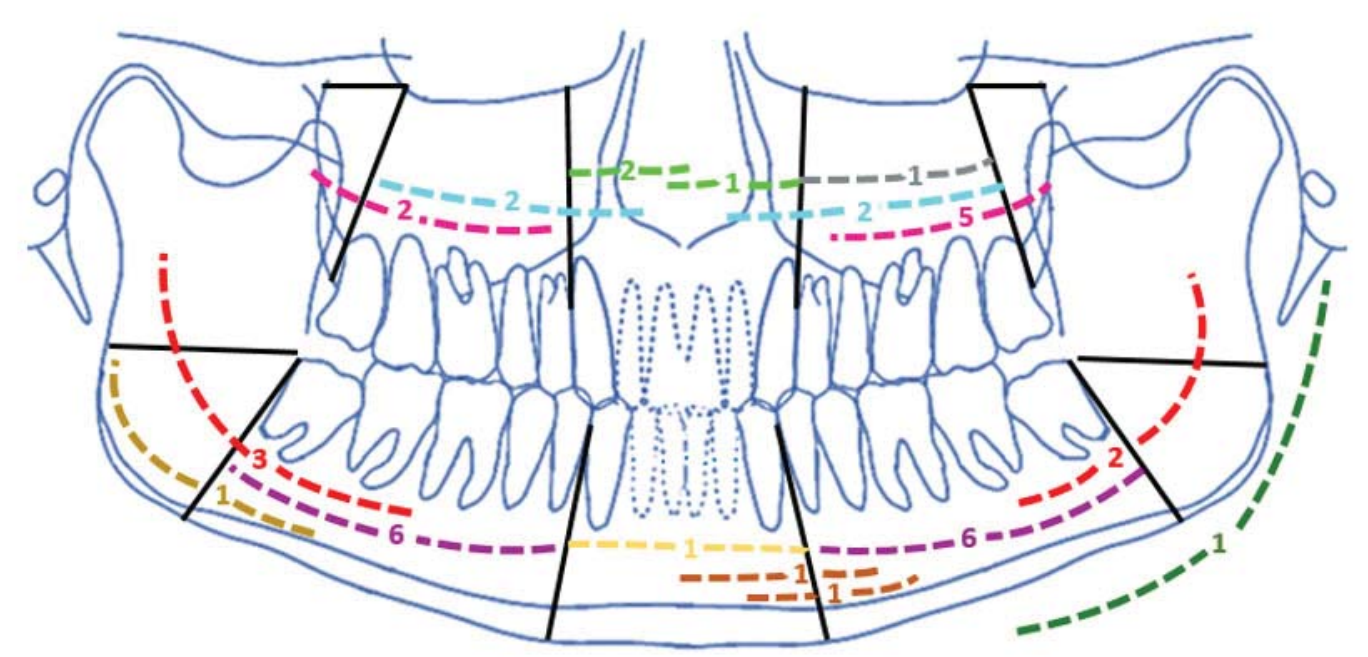

Figura 1. Localización de los quistes dentígeros en los maxilares.

Tabla 2. Distribución según densidad, límites, bordes y configuración interna.

\begin{tabular}{lcc}
\hline Características Radiográficas & n & \% \\
\hline Densidad & 36 & 97,3 \\
Radiolúcido & 1 & 2,7 \\
Mixto & & \\
Límites & 25 & 67,6 \\
Definidos & 11 & 29,7 \\
Parcialmente Definidos & 1 & 2,7 \\
No Definidos & & \\
Bordes & 20 & 54,1 \\
Corticalizado & 11 & 29,7 \\
Parcialmente corticalizado & 5 & 13,5 \\
$\quad$ Recortado & 1 & 2,7 \\
En transición & & \\
Configuración Interna & 35 & 94,6 \\
$\quad$ Unilocular & 2 & 5,4 \\
Multilocular & $\mathbf{3 7}$ & $\mathbf{1 0 0 , 0}$ \\
Total &
\end{tabular}



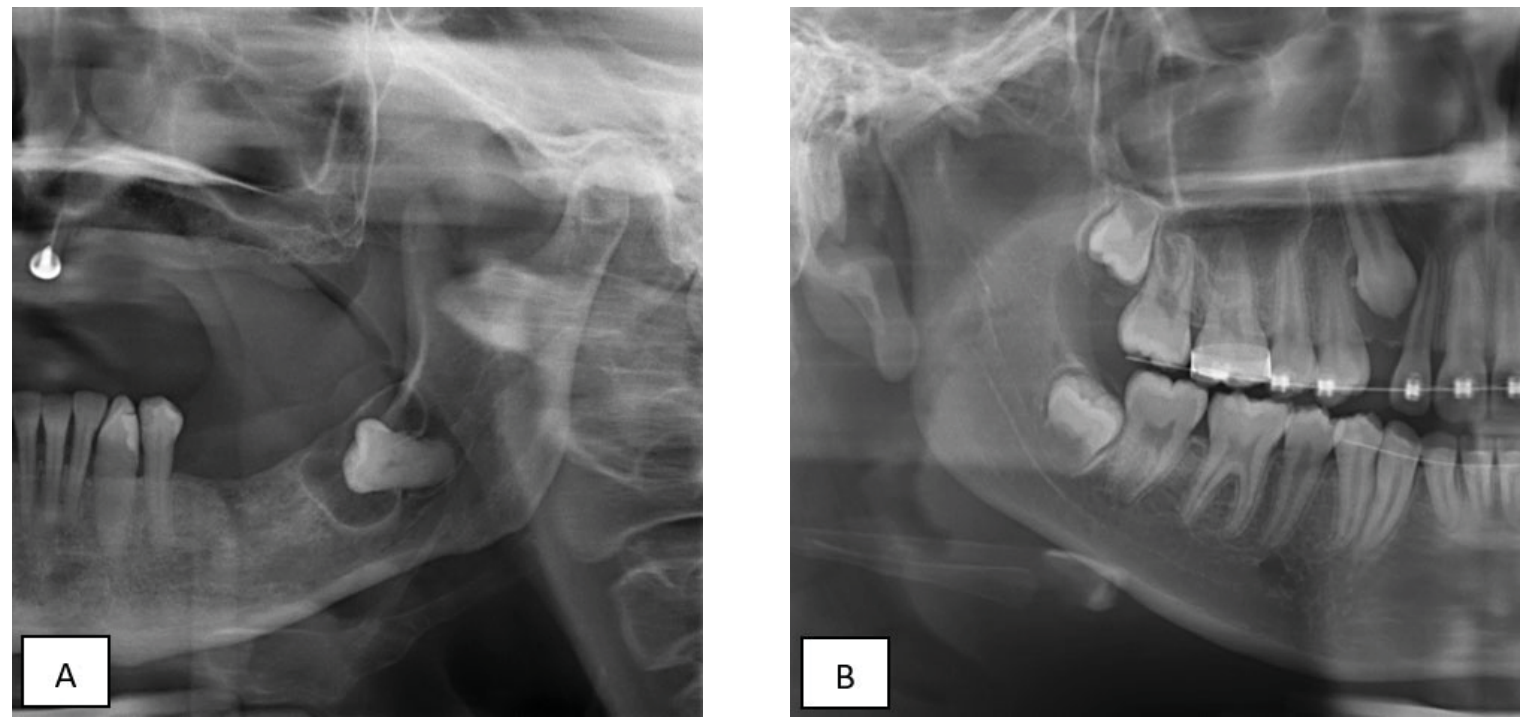

Figura 2. Densidad de la lesión: A) imagen radiolúcida asociada a pieza 38. B) imagen mixta asociada a pieza 13.
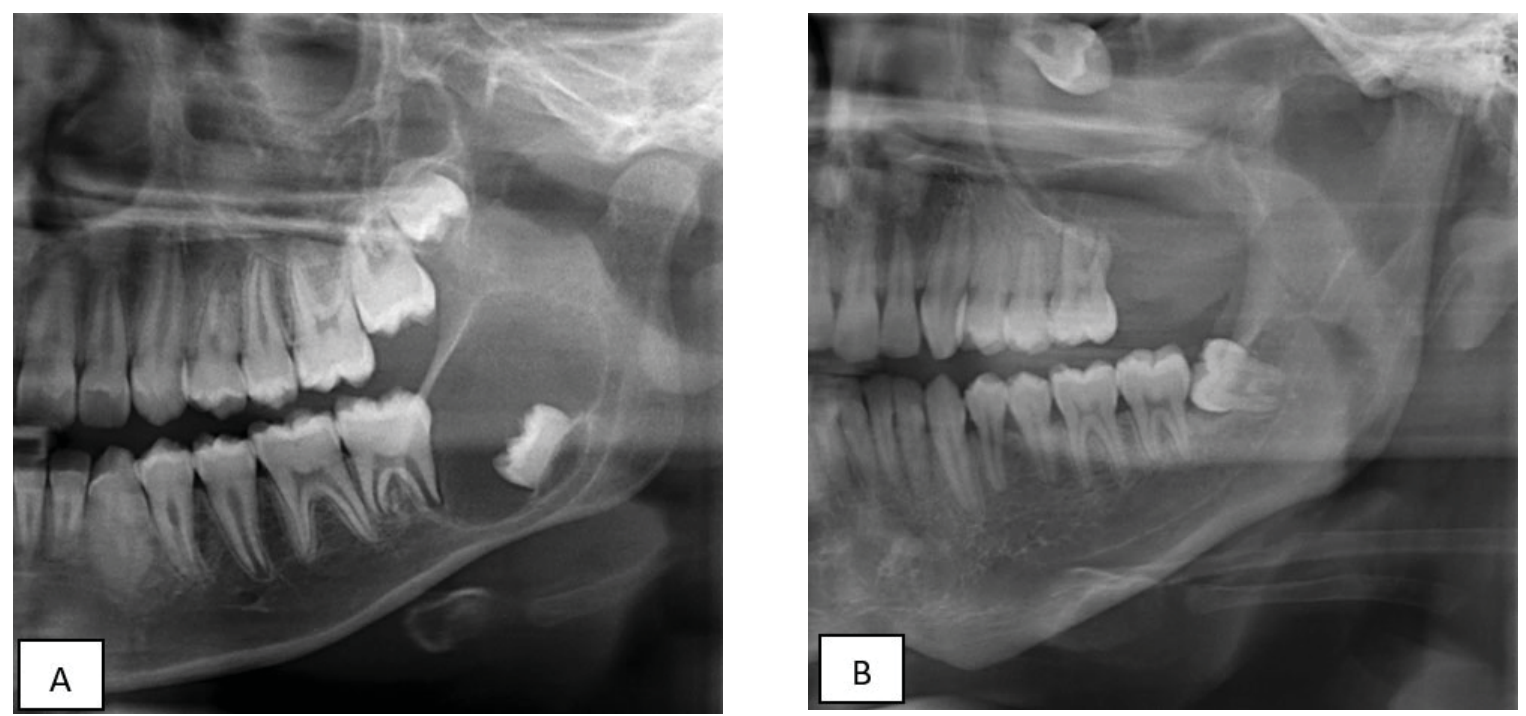

Figura 3. Límites y bordes de la lesión: A) límites definidos, bordes corticalizados. B) límites parcialmente definidos y bordes parcialmente corticalizados. 
Tabla 3. Distribución según la pieza dental asociada.

\begin{tabular}{lcc}
\hline Pieza dental asociada & n & \% \\
\hline 18 & 2 & 5,4 \\
13 & 2 & 5,4 \\
11 & 1 & 2,7 \\
23 & 1 & 2,7 \\
24 & 1 & 2,7 \\
25 & 1 & 2,7 \\
28 & 4 & 10,8 \\
supernumerario superior & 3 & 8,1 \\
38 & 7 & 18,9 \\
37 & 1 & 2,7 \\
35 & 2 & 5,4 \\
43 & 2 & 5,4 \\
45 & 1 & 2,7 \\
46 & 1 & 2,7 \\
47 & 2 & 5,4 \\
48 & 6 & 16,2 \\
Total & $\mathbf{3 7}$ & $\mathbf{1 0 0 , 0}$ \\
\hline
\end{tabular}

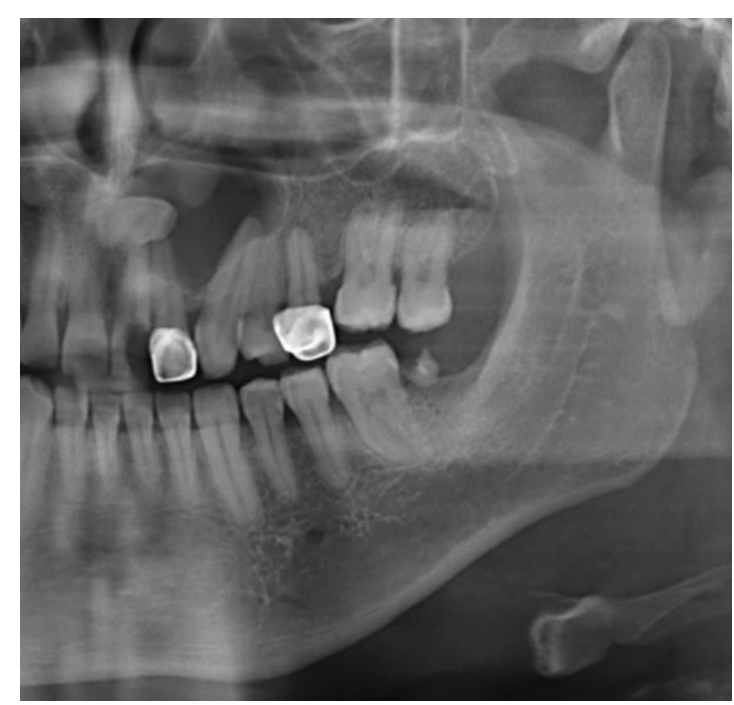

Figura 4. Quiste dentigero asociado a pieza supernumeraria. 
Tabla 4. Distribución según afectación a piezas dentarias y estructura de soporte

\begin{tabular}{lcc}
\hline $\begin{array}{l}\text { Afección a piezas dentarias } \\
\text { estructura de soporte }\end{array}$ & $\mathbf{n}$ & $\boldsymbol{\%}$ \\
\hline Piezas dentarias & & \\
Desplaza & 4 & 10,8 \\
Reabsorbe & 9 & 24,3 \\
Desplaza y reabsorbe & 2 & 5,4 \\
No afecta & 21 & 56,8 \\
No aplica & 1 & 2,7 \\
Lámina dura & & \\
Adelgaza & 3 & 8,1 \\
Reabsorbe & 22 & 59,5 \\
No Afecta & 8 & 21,6 \\
No Registrable & 4 & 10,8 \\
Total & $\mathbf{3 7}$ & $\mathbf{1 0 0 , 0}$ \\
\hline
\end{tabular}
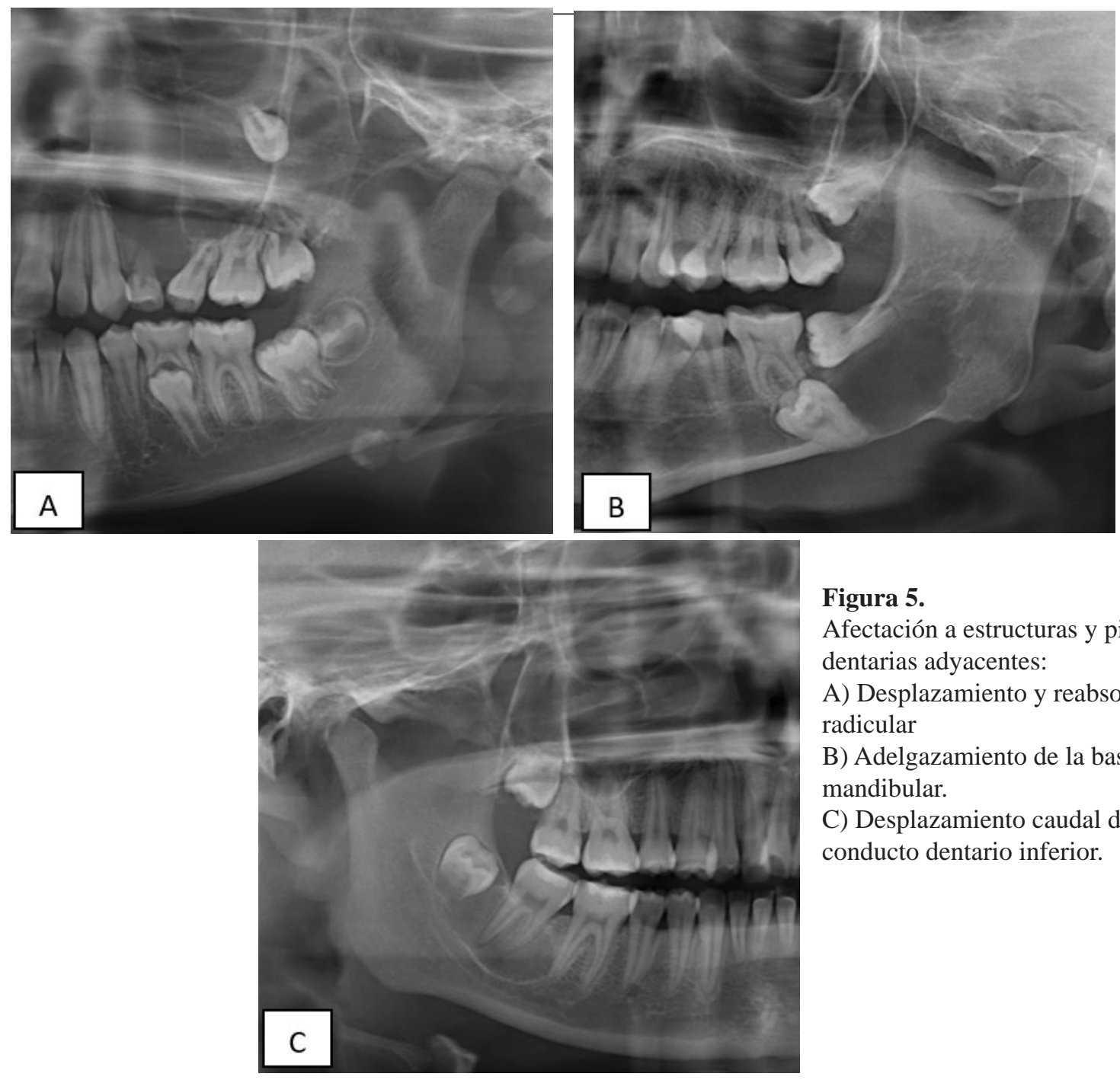

\section{Figura 5.}

Afectación a estructuras y piezas dentarias adyacentes:

A) Desplazamiento y reabsorción radicular

B) Adelgazamiento de la basal mandibular.

C) Desplazamiento caudal del conducto dentario inferior. 
Tabla 5. Distribución según afectación a estructuras óseas del maxilar inferior.

\begin{tabular}{lcc}
$\begin{array}{l}\text { Afección a estructuras óseas del maxilar } \\
\text { inferior }\end{array}$ & n & \% \\
\hline Cortical de la basal mandibular & & \\
Adelgaza & 4 & 18,2 \\
Adelgaza y Reabsorbe & 3 & 13,6 \\
No Afecta & 15 & 68,2 \\
Conducto dentario inferior & & \\
Desplaza & 12 & 54,5 \\
No Afecta & 10 & 45,5 \\
Total & $\mathbf{2 2}$ & $\mathbf{1 0 0 , 0}$ \\
\hline
\end{tabular}

Tabla 6. Casos de quistes dentígeros en distintos países.

\begin{tabular}{|c|c|c|c|c|c|c|c|}
\hline \multirow[t]{2}{*}{ Características } & \multirow{2}{*}{$\begin{array}{l}\text { Número } \\
\text { de casos }\end{array}$} & \multirow{2}{*}{$\begin{array}{c}\text { Promedio } \\
\text { de edad } \\
\text { (años) }\end{array}$} & \multirow{2}{*}{$\begin{array}{l}\text { Rango } \\
\text { (años) }\end{array}$} & \multicolumn{2}{|c|}{ Sexo } & \multicolumn{2}{|c|}{ Localización } \\
\hline & & & & Masculino & Femenino & Maxila & Mandíbula \\
\hline Presente estudio & 37 & 28,1 & $1-80$ & 18 & 19 & 15 & 22 \\
\hline Chile $^{19}$ & 546 & - & $1-90$ & 344 & 202 & 246 & 300 \\
\hline China $^{20}$ & 281 & 11,8 & $1-18$ & 187 & 94 & 160 & 118 \\
\hline *México ${ }^{21}$ & 309 & - & $1-60$ & 191 & 118 & 110 & 159 \\
\hline Portugal $^{22}$ & 28 & 26 & $1-70$ & 17 & 9 & 9 & 19 \\
\hline
\end{tabular}

* 40 casos no especificaron localización

\section{DISCUSIÓN}

En el presente estudio se encontró que el mayor número de casos de quistes dentígeros se ubicaron en la segunda década de vida, dato similar al obtenido por Sridevi et al., (12) y Kambalimath et al., (13), pero diferente al estudio de Demirkol et al.,donde el mayor número de casos de quistes dentígeros se ubicó en la tercera y cuarta década de vida (14). Sin embargo, la Organización Mundial de la Salud (OMS) cita que la incidencia de esta patología se da entre la segunda y cuarta década de vida (15). Este dato es importante y hace deducir que los casos diagnosticados en el Laboratorio de Patología Bucomaxilofacial de la Clínica Dental Docente - UPCH se dieron en personas jóvenes, lo cual tiene un pronóstico favorable, reducien- do o evitando casos de infecciones sobreagregadas o malignizaciones.

Concerniente al sexo, la mayoría de reportes indican que tiene predilección por hombres, como es el caso de países como India (13), México (16)y Taiwan (17). En este estudio se encontró una ligera predilección por mujeres $(51,4 \%)$ esto responde al hecho de que se eliminaron un mayor número de casos de hombres por no cumplir con los criterios de inclusión.

Del total de casos de este estudio el 59,9\% se localizó en la mandíbula, esta predilección por el maxilar inferior concuerda con la mayor parte de la literatura $(14,15)$. Las zonas más afectadas en la mandíbula fueron la zona posterior, seguida de la zona posterior y rama, 
que albergaban el 45,9\% de los casos, esta preferencia por la zona posterior concuerda con la revisión sistemática de 2082 quistes dentígeros de Zhang et al.,(18).

En la tabla 6 se pueden ver las características de los quistes dentígeros en cuanto a edad, sexo y localización más frecuente, comparando la presente investigación con otras investigaciones de distintas partes del mundo.

La gran mayoría de reportes muestran que el quiste dentígero se presenta radiolúcido al igual que la presente investigación (97,3\%), sin embargo, se tuvo un caso donde mostró una pequeña calcificación dentro del universo radiolúcido, por lo que se le consideró mixto, lo cual es poco peculiar y hay escasos reportes acerca de ello, como el de Shibasaki et al., quien reportó un caso donde imagenológicamente el quiste dentígero adoptó un aspecto mixto, el autor menciona que esto pasó debido a la infección del quiste por actinomices, los que penetraron en la lesión por la bolsa periodontal de la pieza asociada y absorbieron calcio y otros minerales del fluido crevicular formando calcificaciones alrededor de la corona del diente incluido (23). Esta causalidad en cuanto a la densidad mixta puede motivar futuras investigaciones.

Numerosos reportes de casos coinciden en que estas lesiones se presentan con límites definidos y bordes corticalizados $(4,8)$; pero cuando estas lesiones se presentan parcialmente definidas o no definidas con bordes parcialmente corticalizadas, recortados o en transición hay que tener en cuenta posibles procesos infecciosos como el presentado por López- Carriches et al., (24), y también posibles malignizaciones como el presentado por Yasuoka et al., (25); el primero evidenció un caso de sinusitis causado por un quiste dentígero infectado, dato importante a tener en cuenta debido a la sintomatología que esta presenta (inflamación de tejido blando, dolor, rinorrea, entre otros). Imagenológicamente se presentó con límites parcialmente definidos y bordes parcialmente corticalizados. El segundo evidenció un caso de carcinoma de células escamosas originado por un quiste dentígero, imagenológicamente se presentó con límites parcialmente definidos y bordes parcialmente corticalizados, siendo la edad más frecuente para estos casos entre la sexta y octava década de vida.
En la revisión sistemática de Bodner et al., mostraron 116 casos de carcinoma intraóseo primario en un periodo de 98 años, menciona que la prevalencia de este tumor derivado de un quiste dentígero se dio en un $16 \%$ (19 casos) (26), no mencionan las características radiográficas, sin embargo, la clasificación del 2017 de la OMS refiere que en etapas avanzadas se muestran con limites poco definidos y bordes no corticalizados pero en etapas tempranas muestran signos radiográficos de benignidad, esto es, limites definidos con bordes corticalizados (27).

La multilocularidad de los quistes dentígeros fue otra característica radiográfica que se evaluó en esta investigación. El resultado obtenido fue similar al hallado por Yoshiura et al., quienes encontraron 2 casos de quistes dentígeros multiloculares en una muestra de 31 casos (28). Así mismo, Scholl et al., mencionaron que esta apariencia radiográfica (lobulada o multilocular) se ve generalmente en quistes dentígeros de gran tamaño; ya que debido a la velocidad desigual de expansión a través de áreas de densidad ósea variables sus bordes adquieren características ondulantes, siendo el aspecto radiográfico resultante comparable a un queratoquiste o ameloblastoma (29). Lo antes dicho se confirma con los dos casos de esta investigación que presentaron aspecto multilocular ya que estas lesiones presentaron gran tamaño y una apariencia similar a queratoquiste o ameloblastoma. Si bien es cierto, estas lesiones usualmente se presentan con apariencia uniquística, sin embargo, cuando estemos frente a una lesión extensa, multilocular y asociada a una pieza dental, debemos considerar también al quiste dentígero como una posibilidad diagnóstica.

Se evaluó también la asociación de piezas dentales con los quistes dentígeros en cuanto al tipo de pieza, formación radicular y desplazamiento de las mismas. Se encontró que el quiste dentígero tiene una marcada predilección por las zonas molares de la mandíbula $(45,9 \%)$, siendo los terceros molares los más afectados, datos similares a los reportados en estudios de poblaciones de Asia (30), América (31) y Europa (32).

En esta investigación, poco más de la mitad de piezas asociadas a la lesión presentaron una formación radicular completa. En la literatura consultada no se encontraron reportes o información sobre esta característica por lo cual no se pudo contrastar con la literatura. 
Otra característica evaluada fue el efecto que los quistes dentígeros pueden tener frente a estructuras como las piezas dentales adyacentes, la cortical de la basal mandibular, el conducto dentario inferior, el piso del seno maxilar y la lámina dura.

La mayor parte de piezas dentales adyacentes $(56,8 \%)$ no se encontraban afectadas (reabsorción radicular o desplazamiento caudal/cefálico) por la lesión quística, pero $24,3 \%$ se encontraban con reabsorción radicular de las mismas, dato significativamente menor al estudio de Struthers et al.,quienes observaron que el quiste dentígero mostró una tendencia a reabsorber raíces $(55 \%)$ y sugieren que la capacidad del quiste dentígero para la reabsorción radicular puede ser el resultado de su origen en el folículo dental, que está asociado con la reabsorción de las raíces de los dientes primarios durante la sucesión normal de los dientes (33).

En cuanto al conducto dentario inferior, 12 casos $(54,5 \%)$ de 22 casos $(100 \%)$ registrados para este efecto; el quiste dentígero hizo contacto con el conducto dentario inferior produciendo su desplazamiento caudal, dato importante a tener en cuenta ya que Liang et al., mencionan que los pacientes con quistes dentígeros mandibulares pueden presentar parestesia del nervio alveolar inferior producido por la alta presión que generan estos quistes o por la corrosión química de los tejidos nerviosos por factores inflamatorios de los quistes (34).

De los quistes dentígeros que afectaron el maxilar superior, se encontró que 7 casos $(46,7 \%)$ desplazaban el piso del seno maxilar y 1 caso $(6,7 \%)$ reabsorbía esta estructura anatómica, no se han encontrado revisiones para conocer y contrastar la prevalencia de esta característica.

Otras características radiográficas evaluadas en el presente estudio fueron la afectación a la basal mandibular y la lámina dura de las piezas adyacentes.

Para el primero, se encontró que de todos los quistes dentígeros presentes en el maxilar inferior (22 casos), el $68,2 \%$ de casos no dañaron la basal mandibular y para el segundo se encontró que de los 37 casos quistes dentígeros, 22 casos $(59,5 \%)$ reabsorbieron la lámina dura de piezas adyacentes. En la literatura no se encontraron datos que nos permitan contrastar ambas características, sin embargo, por los resultados obtenidos en esta investigación, podemos hipotetizar que los quistes dentígeros no tienden a adelgazar y/o reabsorber la cortical de la basal, pero si tienden a reabsorber la cortical de la lámina dura. Este vacío de información podría motivar futuras investigaciones para aceptar o rechazar la hipótesis antes dicha y en cuanto a la cortical de la lámina dura, sería interesante poder evaluarlo mediante otra técnica imagenológica debido a las limitaciones de la radiografía panorámica para tal efecto.

En la literatura consultada no se encontraron revisiones que nos permitan contrastar resultados acerca del desplazamiento de las tablas óseas asociada a quistes dentígeros, sin embargo, se encontraron numerosos reportes de casos como el de Choi et al., (35) Buyukkurt et al., (36) y Freitas et al., (37) donde se observa esta característica.

\section{CONCLUSIONES}

La mayoría de resultados obtenidos sobre las características en población peruana, apoyan estudios previos reportados en América, Europa y Asia. Radiográficamente el quiste dentígero mostró características que avalan su comportamiento netamente benigno.

\section{Correspondencia:}

Maria Alejandra Olaechea Ramos

Correo electrónico: maria.olaechea.r@upch.pe

\section{REFERENCIAS BIBLIOGRAFICAS}

1. Yahara Y, Kubota Y, Yamashiro T, Shirasuna K. Eruption prediction of mandibular premolars associated with dentigerous cyst. Oral Surg Oral Med Oral Pathol Oral Radiol. 2009;108(1):28-31.

2. Qian W, Ma Z, Xie Q, Cai X, Zhang Y, Yang C. Marsupialization facilitates eruption of dentigerous cyst-associated mandibular premolars in preadolescent patient. J Oral Maxillofac Surg. 2013;71(11):1825-32.

3. Shibata Y, Asaumi J, Yanagi Y, et al. Radiographic examination of dentigerous cysts in the transitional dentition. Dentomaxillofac Radiol. 2004;33(1):17-20.

4. Konouchi H, Asaumi J, Yanagi Y, et al. Diagnostic value of MR imaging for dentigerous cysts. Oral Radiol. 2014;30(1):13-19.

5. Devi P, Thimmarasa V, Mehrotra V, Agarwal M. Multiple dentigerous cysts: A case report and review. J Maxillofac Oral Surg. 2015;14(1):47-51. 
6. Hedge R, Khare S, Deyrukhkar V. Dentigerous cyst in a young child: Clinical insight and a case report. $\mathrm{J}$ Indian Soc Pedod Prev. 2013;31(1):209-11.

7. Zapala-Póspiech A, Wyszynska-Pawelec G, Adamek D, Tomaszewska R, Zaleska M, Zapata J. Malignant transformation in the course of a dentigerous cyst: A problem for a clinician and a pathologist: Considerations based on a case report. Pol J Pathol. 2013;64(1):65-8.

8. Jeon J, Park C, Cho S, Hwang K. Bilateral dentigerous cyst that involve all four dental guadrants: A case report and literature review. J Korean Assoc Oral Maxillofac Surg. 2016;42(2):123-6.

9. Langlais R, Langland O, Nortjé C. Diagnostic imaging of the jaws. Malvern. Williams and Wilkins; 1995.

10. Albarrán G, Gutierrez R, Yépez Y. Marsupialización como tratamiento para quiste dentígero en un paciente pediátrico. Reporte de caso. Rev Odontol Andes. 2014;9(1):47-55.

11. Kouhsoltani M, Mesgarzadeh A, Moradzadeh M. Mandibular fracture associated with a dentigerous cyst: Report of a case and literatura review. J Dent Res Dent Clin Dent Prospects. 2015;9(3):193-8.

12. Sridevi K, Kaushik A, Ramaswamy P, Manjula M, Vinod V, Aravinda K. Dentigerous cysts of maxilofacial región-clinical, radiographic and biochemical analysis. Kathmandu Univ Med J. 2015;13(49):8-11.

13. Kambalimath D, Kambalimath H, Agrawal S, et al. Prevalence and distribution of odontogenic cyst in Indian population: a 10-year retrospective study. J Maxillofac Oral Surg. 2014;13(1):10-5.

14. Demirkol M, Ege B, Yanik S, Aras M, Ay S. Clinicopathological study of jaw cysts in southeast región of Turkey. Eur J Dent. 2014;8(1):107-11. doi: 10.4103/1305-7456.126260.

15. Speight P, Fantasia J, Neville B. Odontogenic and non-odontogenic developmental cysts. Dentigerous cyst. En: El Naggar A, Chan J, Grandis J, Takata T, Slootweg P. (Editors). WHO Classification of Head and Neck Tumours. 4th Ed. Lyon: WHO; 2017. p. 234.

16. Mosqueda A, Irigoyen M, Díaz M, Torres M. Quistes odontogénicos. Análisis de 856 casos. Medicina Oral. 2002:7(1):89-96.

17. Lin H, Wang Y, Chen H, Cheng S, Sun A, Chiang C. A clinicopathological study of 338 dentigerous cysts. J Oral Pathol Med. 2013;42(6):462-7.

18. Zhang L, Yang R, Li W. Macdonald-Jankowski D. Poh C. Dentigerous cyst: A retrospective clinicopathology analysis of 2082 dentigerous cysts British Columbia, Canada. Int J Oral Maxillofac Surg. 2010; 39(9): 878-82.

19. Ochsenius G, Escobar E, Godoy L, Peñafiel C. Odontogenic cysts: analysis of 2944 cases in Chile. Med Oral Pathol Oral Cir Bucal. 2007;21(1):85-91.
20. Li N, Gao X, Xu Z, et al. Prevalence of developmental odontogenic cysts in children and adolescents with emphasis on dentigerous cyst and odontogenic keratocyst (keratocyst odontogenic tumor). Acta Odontol Scand. 2014;72(8):795-800.

21. Villasis L, Portilla J, Melendez A, Gaitan L, Leyva E. Prevalence and distribution of odontogenic cysts in a Mexican simple: A 753 cases study. J Clin Exp Dent. 2017;9(4):531-38.

22. Monteiro L, De la Peña J, Fonseca L, Paiva A, Do Amaral B. Odontogenic cysts-A descriptive clinicopathological study. Braz J Oral Sci. 2005;4(12):670-75.

23. Shibasaki M, Iwai T, Chikuma H, Mitsudo K, Inayama Y, Tohnai I. Actinomyces associated calcifications in a dentigerous cyst of the mandible. J Craniofac Surg. 2013;24(3):311-4.

24. Lopez-Carriches C, Lopez-Carriches I, Bryan R. Odontogenic sinusitis caused by an inflammation of a dentigerous cyst and subsequent finding of a fibrous displasia. A case report. Open Dent J. 2016;30(10):647-55.

25. Yasuoka T, Yonemoto K, Kato Y, Tatematsu N. Squamous cell carcinoma arising in a dentigerous cyst. $\mathrm{J}$ Oral Maxillofac Surg. 2000; 58(8):900-5.

26. Bodner L, Manor E, Shear M, Van Der Waal I. Primary intraosseous squamous cell carcionma arising in an odontogenic cyst: a clinicopathologic analysis of 116 reported cases. J Oral Pathol Med. 2011;40(10):733-8.

27. Speight P, Fantasia J, Neville B. Odontogenic and non-odontogenic developmental cysts. Dentigerous cyst. En: El Naggar A, Chan J, Grandis J, Takata T, Slootweg P. WHO Classification of Head and Neck Tumours. 4th Ed. Lyon: WHO; 2017. p. 234.

28. Yoshiura K, Higuchi Y, Araki K, et al. Morphologic analysis of odontogenic cysts with computed tomography. Oral Surg Oral Med Oral Pathol Oral Radiol Endod. 1997;83(6):712-8.

29. Scholl R, Kellett H, Neumann D, Lurie A. Cysts and cystic lesions of the mandible: Clinical and radiologic histopathologic review. Radiographics. 1999;19(5):1107-24.

30. Yeo J, Rosnah B, Ti L, Zhao Y, Ngeow W. Clinipathological study of dentigerous cysts in a Singapore and Malaysia. Malays J Pathol. 2007;29(1):41-7.

31. Avelar R, Antunes A, Carvalho R, Bezerra P, Oliveira Neto P, Andrade E. Odontogenic cysts: A clinicopathological study of 507 cases. J Oral Sci. 2009;51(4):581-86.

32. Henien M, Sproat C, Kwok J, Beneng K, Patel V. Coronectomy and dentigerous cysts: a review of 68 patients. Oral Surg Oral Med Oral Pathol Oral Radiol. 2017;123(6):670-74.

33. Struthers P, Shear M. Root resorption by ameloblastomas and cyst of the jaws. Int J Oral Surg. 1976;5(3):128-32. 
34. Liang $\mathrm{Y}, \mathrm{He} \mathrm{W}$, Zheng $\mathrm{P}$, Liao G. Inferior alveolar nerve function recovers after descompression of large mandibular cystic lesions. Oral Dis. 2015;21(5):674-8.

35. Choi H, Lee J. Obliteration of recurrent large dentigerous cyst using bilateral buccal fat pad sling flaps. J Craniofac Surg. 2016;27(5):465-8.

36. Buyukkurt M, Omezli M, Miloglu O. Dentigerous cyst associated with an ectopic tooth in the maxillary sinus: a report of 3 cases and review of the literatura. Oral Surg Oral Med Oral Pathol Oral Radiol Endod. 2010;109(1):67-71.

37. Freitas D, Tempest L, Sicoli E, Lopes-Neto F. Bilateral dentigerous cysts: Review of the literature and report of an unusual case. Dentomaxillofac Radiol. 2006;35(6):464-8.

Recibido: 23-08-2018

Aceptado: 14-11-2018 